\title{
DIAMETER-PRESERVING LINEAR BIJECTIONS OF FUNCTION SPACES
}

\author{
T. S. S. R. K. RAO and A. K. ROY \\ (Received 28 October 1999; revised 1 November 2000)
}

Communicated by P. G. Dodds

\begin{abstract}
In this paper we give a complete description of diameter-preserving linear bijections on the space of affine continuous functions on a compact convex set whose extreme points are split faces. We also give a description of such maps on function algebras considered on their maximal ideal space. We formulate and prove similar results for spaces of vector-valued functions.
\end{abstract}

2000 Mathematics subject classification: primary 46J10, 46E40, 47B38.

\section{Introduction}

Let $X$ be a compact Hausdorff space and let $C(X)$ denote the space of complexvalued continuous functions equipped with the supremum norm. Let $A \subset C(X)$ be a closed subspace containing constants and separating points of $X$. A linear bijection $\Phi$ of $A$ is said to be diameter-preserving if $\operatorname{diam}(\Phi(f)(X))=\operatorname{diam}(f(X))$ for every $f \in A$. When $A=C(X)$ and $X$ a first countable space, Györy and Molnar [GM] have given a complete description of diameter-preserving linear bijections in terms of homeomorphisms of $X$ and linear functionals on $C(X)$. Their result has been extended to the case of a general compact Hausdorff space by González and Uspenskij [GU] and independently by Cabello Sanchez [C].

Motivated by this, in this paper we study diameter-preserving linear bijections between function spaces and spaces of vector-valued continuous functions. Our approach follows closely that of González and Uspenskij [GU]. For a function space $A$ that separates points of $X$ and contains constants, let $S_{A}$ denote the state space of $A$,

(C) 2001 Australian Mathematical Society 0263-6115/2001\$A2.00+0.00 
that is, $S_{A}=\left\{\Lambda \in A^{*}: \Lambda(1)=1=\|\Lambda\|\right\}$. We first consider the situation when $A$ is a self adjoint subspace of $C(X)$ (that is, $f \in A \Rightarrow \bar{f} \in A$ ). In this case it is well known and easy to see that $A$ is isometric to $A\left(S_{A}\right)$, the space of affine continuous functions ( $S_{A}$, as usual, has the weak* topology of $A^{*}$ ). Thus in the first part of the paper, for a compact convex set $K$, we consider the space $A(K)$ and diameter preserving linear bijections on $A(K)$. Let $\partial_{e} K$ denote the set of extreme points of $K$. We give a complete description of diameter-preserving linear bijections, under the hypothesis that every point of $\partial_{e} K$ is a split face (see [AE] for the notion of the split face and for other concepts from convexity theory that we will be using in this paper). We note that this hypothesis is satisfied when $K$ is a Choquet simplex (which is the case when $K=S_{A}$ and $A=C(X)$ ) and also when $A$ is a subalgebra (see [AE, Chapter 3 and Theorem 4.4 on page 168]). Thus our results extend those of Györy and Molnar, González and Uspenskij.

We now give a more detailed description of our results. As in the case of Györy and Molnar, our work is in part motivated by the Banach-Stone type theorems in the literature that describe isometries of function spaces. See $[L, R 1, B]$ and the survey article [FJ].

For a compact convex set $K$ and an affine homeomorphism $\phi$ of $K$, for any $t \in \Gamma$ (unit circle) and for any $\Lambda \in A(K)^{\prime}$ (space of linear functionals) such that $\Lambda(1) \neq-t$, it is easy to see that $a \rightarrow t a \circ \phi+\Lambda(a) 1$ is a diameter-preserving linear bijection of $A(K)$. In the first part of this paper, under the additional hypothesis that every extreme point of $K$ is a split face, we show that any diameter-preserving linear bijection is of the above form. We give an example to show that the additional hypothesis we are assuming is not a necessary condition.

We next show that if $A$ is a function algebra with maximal ideal space $X$, then again a description similar to the one given above is available for the diameter-preserving linear bijections. We also note that for a metric space $X$ that is topologically complete, the diameter-preserving linear bijections of $C_{b}(X)$, the space of bounded continuous functions on $X$, can also be described via homeomorphisms of $X$.

For a Banach space $E$, let $C(X, E)$ denote the space of $E$-valued continuous functions an $X$, equipped with the supremum norm. Analogous to the scalar valued case let $\phi$ be a homeomorphism of $X$, let $S$ be an onto isometry of $E$ and $T$ : $C(X, E) \rightarrow E$ be a linear map such that $S+T / 1 \otimes E$ is a bijection. One can show that $\Phi(f)(x)=S(f(\phi(x)))+T(f)$ is a diameter-preserving linear bijection of the space $C(X, E)$. We recall that a linear projection $P$ on $E$ is said to be an $L$-projection, if $\|P(e)\|+\|(e-P(e))\|=\|e\|$ for all $e \in E$. The range of an $L$-projection is called an $L$-summand.

In the concluding part of the paper we show that if for all $f \in \partial_{e} E_{1}^{*}$, line $\{f\}$ is an $L$-summand, then any diameter-preserving linear bijection of $C(X, E)$ is of the above form. We also consider the space of functions taking values in a dual space 
equipped with the weak ${ }^{*}$ topology as well as spaces of vector-valued affine continuous functions.

\section{Function spaces}

Let $K_{1}$ and $K_{2}$ be two compact convex sets. A linear bijection $\Phi: A\left(K_{1}\right) \rightarrow A\left(K_{2}\right)$ is said to be diameter-preserving if for all $a \in A\left(K_{1}\right)$

$$
\sup _{x, y \in K_{1}}|a(x)-a(y)|=\sup _{x, y \in K_{2}}|\Phi(a)(x)-\Phi(a)(y)| .
$$

Note that since $a$ and $\Phi(a)$ are affine continuous, this is equivalent to

$$
\sup _{x, y \in \partial_{e} K_{1}}|a(x)-a(y)|=\sup _{x, y \in \partial_{e} K_{2}}|\Phi(a)(x)-\Phi(a)(y)| .
$$

Thus in order to obtain a description of $\Phi$ analogous to the description in the case of space of continuous functions, it is natural to impose additional condition involving extreme points.

In what follows we consider a compact convex set $K$ as canonically embedded in $A(K)^{*}$ via evaluation mapping. We note that $A(K)_{1}^{*}=\left\{\Lambda \in A(K)^{*}:\|\Lambda\| \leq 1\right\}=$ $\overline{c o}\left(\Gamma \partial_{e} K\right)$ and $\partial_{e} A(K)_{1}^{*}=\Gamma \partial_{e} K$. (Here $\Gamma$ denotes the unit circle, co denotes the convex hull and the closure is taken with respect to the weak*-topology.)

Following the notation of [GU] we denote by

$$
T=\left\{\alpha(x-y): x, y \in \partial_{e} K \text { and } \alpha \in \Gamma\right\} .
$$

Thus $\{a \in A(K): \operatorname{diam}(a(K)) \leq 1\}^{0}=\overline{\mathrm{co}}(T)$.

LEMMA 1. Suppose every element of $\partial_{e} K$ is a split face. Then $\partial_{e} \overline{c o}(T)=T \backslash\{0\}$.

PROOF. We first show that for $x \neq y$ in $\partial_{e} K, x-y \in \partial_{e} \overline{c o}(T)$. As in the proof of Lemma in [GU], it is enough to show that $x-y$ is an extreme point of the 2-ball $\left\{\Lambda \in A(K)^{*}:\|\Lambda\| \leq 2, \Lambda(1)=0\right\}$. Since $x$ and $y$ are disjoint split faces, it follows from [AE, Theorem 4.4, page 168] that for any complex numbers $\alpha$ and $\beta$, $\|\alpha x+\beta y\|=|\alpha|+|\beta|$. Thus by arguments identical to the ones given in [GU] we conclude that $x-y \in \partial_{e} \overline{c o}(T)$.

Conversely by an application of Milman's theorem [D, Chapter IX, Corollary 4] we have, $\partial_{e} \overline{\operatorname{co}}(T) \subset\left\{\alpha(x-y): x, y \in \overline{\partial_{e} K}\right.$ and $\left.\alpha \in \Gamma\right\}$. Let $x \neq y, x, y \in \overline{\partial_{e} K}$ and $x-y \in \partial_{e} \overline{c o}(T)$. Suppose $x \notin \partial_{e} K$ and let $x=\lambda x^{1}+(1-\lambda) x^{2}$ for $x^{1} \neq x^{2} \in K$ and $0<\lambda<1$. Now $x-y=\lambda\left(x^{1}-y\right)+(1-\lambda)\left(x^{2}-y\right)$. Since $y \in \overline{\partial e K}$, there exists a net $\left\{y_{\alpha}\right\} \subset \partial_{e} K$ such that $y_{\alpha} \rightarrow y$. Similarly, we can find nets of convex combinations of vectors in $\partial_{e} K$ that converge to $x^{1}$ and $x^{2}$ respectively. Therefore, 
$x^{1}-y, x^{2}-y \in \overline{c o}(T)$. This contradicts our hypothesis $x-y \in \partial_{e} \overline{\operatorname{co}}(T)$. Hence $x, y \in \partial_{e} K$.

For any $\alpha_{0} \in \Gamma, x_{0} \in \partial_{e} K, A=\left\{\alpha\left(x_{0}-y\right): y \in \partial_{e} K\right\}$ is such that $A-A \subset T$. Our next lemma shows that under our hypothesis this is a superset of such subsets of $T$.

LEMMA 2. Suppose every element of $\partial_{e} K$ is a split face. Let $A \subset T$ be a set having more than two points and such that $A-A \subset T$. Then there exists a unique $x_{0} \in \partial_{e} K$ and $\alpha \in \Gamma$ such that $A \subset\left\{\alpha\left(x_{0}-y\right): y \in \partial_{e} K\right\}$.

PROOF. That the lemma is false if $|A|=2$ is seen by taking $A=\left\{e^{i t}(x-\right.$ $\left.y), e^{i t+i \pi / 3}(x-y)\right\}$, where $x, y \in \partial_{e} K$. For the remainder of the proof, we rely on the well-known fact that line $\{x\}$ is an $L$-ideal in $A(K)^{*}$ whenever $x \in \partial_{e} K$ (see [AE, Theorem 4.4, page 168]).

Let $p_{1}=\alpha(x-y)$ and $p_{2}=\beta(u-v) \in A$ be distinct, with $p_{1}-p_{2} \in T$. Thus $\|\alpha(x-y)-\beta(u-v)\|=2$. If $\{x, y\} \cap\{u, v\}$ is empty then, since they are all distinct, $\|\alpha(x-y)-\beta(u-v)\|=4$, a contradiction. Thus $|\{x, y\} \cap\{u, v\}|=2$ or 1 . The first case is easily disposed off in as much as $p_{1}=e^{i t}(x-y), p_{2}=e^{i(t+\pi / 3)}(x-y)$ and any $p_{3}$ be of the form $e^{i(t+2 \pi / 3)}(x-y)$ or $e^{i(t+\pi / 3)}(x-z)$ or $e^{i t}\left(x-y^{\prime}\right)$ and so on will give a contradiction, as the calculations that follow will show.

In the second case, we have the possibilities: $u=x$ or $u=y, v=x$ or $v=y$. Considering $u=x$, we have

$$
\|\alpha(x-y)-\beta x+\beta v\|=\|(\alpha-\beta) x-\alpha y+\beta v\|=|\alpha-\beta|+2=2 .
$$

Thus $\alpha=\beta$ and $p_{2}=\alpha(x-v)$. The other cases are similarly dealt with to yield that $\alpha=\beta$ and $p_{2}=\alpha(x-v)$ or $p_{2}=\alpha(u-y)$. The rest of the proof follows [GU] to yield the desired conclusion.

Now we are ready to give a complete description of diameter-preserving linear bijections between $A(K)$ spaces. Let line $\{1\}$ denote the constant functions in $A(K)$.

THEOREM 1. Let $K_{1}$ and $K_{2}$ be two compact convex sets such that every extreme point is a split face. Let $\Phi: A\left(K_{1}\right) \rightarrow A\left(K_{2}\right)$ be a diameter-preserving linear bijection. Then there exists an affine homeomorphism $\phi: K_{2} \rightarrow K_{1}, \Lambda \in A\left(K_{1}\right)^{\prime}$ and $\alpha_{0} \in \Gamma$ such that $\Phi(a)=\alpha_{0} a \circ \phi+\Lambda(a) 1$ for any $a \in A\left(K_{1}\right)$.

Proof. Let $E$ and $F$ denote respectively, the quotient spaces $A\left(K_{1}\right) /$ line $\{1\}$ and $A\left(K_{2}\right) /$ line $\{1\}$. Let $\tilde{\Phi}$ be the natural extension of $\Phi$ to the quotient spaces. As in the proof of [GU, Theorem 5.1], it is easy to see that $\tilde{\Phi}$ is a well defined, linear, bi-continuous, bijection. As $\Phi$ is a diameter-preserving map, in terms of our earlier 
notation, since $(\tilde{\Phi})^{*}$ is an affine homeomorphism such that $(\tilde{\Phi})^{*}\left(\overline{\mathrm{co}}\left(T_{2}\right)\right)=\overline{\mathrm{co}}\left(T_{1}\right)$, we get $(\tilde{\Phi})^{*}\left(T_{2}\right)=T_{1}$.

Fix $x_{0} \in \partial_{e} K_{2}$ and define $g(x)=(\tilde{\Phi})^{*}\left(x-x_{0}\right), x \in \partial_{e} K_{2}$.

Note that $g\left(x_{1}\right)-g\left(x_{2}\right)=(\tilde{\Phi})^{*}\left(x_{1}-x_{2}\right) \in T_{1}$ for any $x_{1}, x_{2} \in \partial_{e} K_{2}$. Thus if $A=g\left(\partial_{e} K_{2}\right)$, then $A-A \subset T_{1}$. It therefore follows from Lemma 2 that there is a unique $y_{0} \in \partial_{e} K_{1}$ and $\alpha_{0} \in \Gamma$ such that

$$
A \subset\left\{\alpha_{o}\left(y-y_{0}\right): y \in \partial_{e} K_{1}\right\} .
$$

We now claim that $A=\left\{\alpha_{0}\left(y-y_{0}\right): y \in \partial K_{1}\right\}$. Let $y \in \partial_{e} K_{1}$, since $\alpha_{0}\left(y-y_{0}\right) \in$ $T_{1}=(\tilde{\Phi})^{*}\left(T_{2}\right)$, there exists $x_{1}, x_{2} \in \partial_{e} K_{2}$ and $\beta \in \Gamma$ such that

$$
\begin{aligned}
\alpha_{0}\left(y-y_{0}\right) & =(\tilde{\Phi})^{*}\left(\beta\left(x_{1}-x_{2}\right)\right)=(\tilde{\Phi})^{*}\left(\beta\left(x_{1}-x_{0}\right)\right)-(\tilde{\Phi})^{*}\left(\beta\left(x_{0}-x_{2}\right)\right) \\
& =\beta\left(g\left(x_{1}\right)-g\left(x_{2}\right)\right)=\beta \alpha_{0}\left(y^{\prime}-y^{\prime \prime}\right)
\end{aligned}
$$

for some $y^{\prime}, y^{\prime \prime} \in \partial_{e} K_{1}$.

Therefore, by arguments similar to the ones given during the proof of Lemma 2, we get that $\beta=1, y^{\prime}=y, y^{\prime \prime}=y_{0}$ or $\beta=-1, y^{\prime}=y_{0}, y^{\prime \prime}=y$. Clearly in either case $\alpha_{0}\left(y-y_{0}\right) \in g\left(\partial e K_{2}\right)=A$.

Hence $A=\left\{\alpha_{0}\left(y-y_{0}\right): y \in \partial_{e} K_{1}\right\}$. Define $\phi: \partial_{e} K_{2} \rightarrow \partial_{e} K_{1}$ by $\phi(x)=$ $\bar{\alpha}_{0} g(x)+y_{0}$. Since $(\tilde{\Phi})^{*}$ is a linear bijection and weak* homeomorphism, it is easy to see that $\phi$ can be extended to an onto affine homeomorphism, still denoted by $\phi$, between $K_{2}$ and $K_{1}$ such that $\phi(x)=\bar{\alpha}_{0}(\tilde{\Phi})^{*}\left(x-x_{0}\right)+y_{0}$ for $x \in K_{2}$. Therefore, as in the proof of [GU, Theorem 5.1] we conclude that there exists a $\Lambda \in A\left(K_{1}\right)^{\prime}$ such that

$$
\Phi(a)=\alpha_{0} a \circ \phi+\Lambda(a) \text { for all } a \in A\left(K_{1}\right) .
$$

REMARK 1. It would be interesting to know if the above result can be proved by assuming the split face hypothesis only on $K_{1}$.

We next consider the description of diameter-preserving linear bijection for the case of function algebras. Here we could prove the result in full generality under the additional hypothesis that $X$ is the maximal ideal space of $A$. We also discuss some special cases where one can dispense away with this assumption.

As noted in the Introduction, if $S_{A}$ denotes the state space of a function algebra $A$, then every extreme point of $S_{A}$ is a split face or in the terminology of function algebras, every extreme point is a generalized peak point (see [AE, Corollary 4.3, page 166]). If $\Phi: A \rightarrow A$ is a linear diameter-preserving bijection, then by invoking [AE, Theorem 4.4, page 168] and using arguments similar to the ones indicated before one can get an onto homeomorphism $\phi: \overline{\partial_{e} S_{A}} \rightarrow \overline{\partial_{e} S_{A}}, \alpha \in \Gamma$ and $\Lambda \in A^{\prime}$ such that $\Phi(f)=\alpha f \circ \phi+\Lambda(f)$ on $\overline{\partial_{e} S_{A}}$. (We recall that $\overline{\partial_{e} S_{A}}$ is called the Shilov 
boundary of $A$.) However, in general, $\phi$ cannot be extended to a homeomorphism of the underlying space $X$ so that the above equation is valid on all of $X$.

EXAmple 1. Let $X=\{z: 1 / 2 \leq|z| \leq 1\}$ and $D=\{z:|z| \leq 1\}$. Let $B$ be the disc algebra on $D$ (that is, closure of the space of polynomials) and let $A=B / X$. For this function algebra it is easy to see that $\Gamma$ is the Shilov boundary of $A$. Hence $\phi(t)=(t-1 / 2) /(1-t / 2)$ is a homeomorphism of $\Gamma$ which does not have an extension to $X$.

In the following proposition we overcome this difficulty by assuming that $X$ is the maximal ideal space of $A$ (thus identity is the Gelfand embedding).

PROPOSITION 1. Consider $A$ as a function algebra on its maximal ideal space $X$. Let $\Phi$ be a diameter-preserving linear bijection of $A$. Then there exists an onto homeomorphism $\tilde{\phi}$ of $X, a \alpha \in \Gamma$ and $a \Lambda \in A^{\prime}$ such that $\Phi(a)=\alpha a \circ \tilde{\phi}+\Lambda(a)$ for all $a \in A$.

PROOF. In view of the above discussion, we only need to show that the homeomorphism $\phi$ from the Shilov boundary can be extended to a homeomorphism $\tilde{\phi}$ of $X$. For $x \in X$, let us consider the complex functional $\tilde{\phi}(x)$ defined on $A$ by

$$
\tilde{\phi}(x)(a)=f(x),
$$

where $f \in A$ is such that $f=a \circ \phi$ on the Shilov boundary. Clearly, $\tilde{\phi}(x)$ is a homomorphism and hence defines an element of $X$. It is easy to see that $x \rightarrow(\tilde{\phi})(x)$ is continuous (as the weak topology defined by $A$ agrees with that of $X$ ), one-to-one, onto and is an extension of $\phi$. Thus $\Phi$ has the required form.

\section{Some examples}

By way of illustrating the general results proved thus far, we describe the diameterpreserving linear bijections in some concrete function spaces and algebras. We also give an example to show that it is not a necessary condition for the validity of Theorem 1 that extreme points are split faces.

(1) The disc algebra $A(D)$.

If $D=\{z:|z| \leq 1\}$, then $A(D)=\{f \in C(D): f$ is analytic in the interior of $D\}$, with the supremum norm, is the traditional disc algebra. By the maximum modulus theorem, the Shilov boundary of $A(D)$ is $\Gamma=\{z:|z|=1\}$. The maximal ideal space of $A$ is $D$ itself. We know from Proposition 1 that a diameter-preserving linear bijection is given by a homeomorphism $h: D \rightarrow D$ such that $f \circ h \in A(D)$ for all 
$f \in A(D)$. In particular, take $f$ to be the identity function $z$ on $D$ to conclude that $h \in A(D)$. Similarly, $h^{-1} \in A(D)$ and since $h \circ h^{-1}(z)=z$ for all $z \in D$, we see that $h$ is a conformal automorphism of $D$. Thus a linear diameter-preserving bijection $\Psi: A(D) \rightarrow A(D)$ looks like

$$
\Psi(f)(z)=\alpha f \circ \psi_{a}(z)+c(f),
$$

where

$$
\psi_{a}(z)=\frac{(z-a)}{1-\bar{a} z}, \quad(|a|<1,|\alpha|=1 \text { and } c(f) \in A(D))
$$

(2) The space of bounded continuous functions.

Let $C_{b}(X)$ denote the space of bounded continuous functions on a completely regular space $X$, equipped with the supremum norm. One knows that this space can be realized isometrically as $C(\beta(X))$, where $\beta(X)$ is the Stone-Čech compactification of $X$. The notion of a diameter-preserving linear bijection on $C_{b}(X)$ can be defined in the usual way, and it is obvious that it is given essentially by a homeomorphism $h$ of $\beta(X)$ onto itself. But since we would like the answer to be in terms of $X$, we have to decide when $h$ takes $X$ onto $X$.

Assume now that $X$ is a metric space that is topologically complete. Then any point of $X$ is a $G_{\delta}$ set in $\beta(X)$. According to [GJ, Corollary 9.2, page 132], no point of $\beta(X) \backslash X$ can be a $G_{\delta}$. Thus a homeomorphism of $\beta(X)$ will carry $X$ onto itself. Also when $X$ is a $G_{\delta}$ subset of $\beta(X) \backslash X$ such that every point of $X$ is the limit of a sequence of distinct points, then by applying [W, Proposition 2.12, page 53], we again have that $h$ maps $X$ onto itself.

(Thanks are due to our colleague $\operatorname{Dr}$ A. B. Raha, for some helpful conversations on this topic.)

We conclude this section by giving an example to show that it is not a necessary condition for the validity of Theorem 1 that every extreme point is a split face. We consider the real scalar field.

(3) Affine functions on the square.

Let $S$ be the square in the plane with vertices at the points, $x_{1}=(1,1), x_{2}=(-1,1)$, $x_{3}=(-1,-1)$ and $x_{4}=(1,-1)$. Clearly none of the extreme points are split faces and also $x_{i}-x_{j}$ is not an extreme point of the two ball in $A(S)^{*}$ for $1 \leq i, j \leq 4$. We now show that every diameter-preserving linear bijection of $A(S)$ is induced by an affine homeomorphism of $S$.

Let us first observe that it is enough to prove this only for diameter-preserving linear bijections $D$ of $A_{0}(S)=\{f \in A(S): f(0)=0\}$. Any $f \in A_{0}(S)$ is of the form $a x+b y,\|f\|=\max \{|a+b|,|a-b|\}$. Clearly, $D$ can be represented by a $2 \times 2$ matrix say $D=\left(a_{i j}\right)$. Since $D$ is diameter-preserving, using successively the 
functions, $(1,0),(0,1),(1,-1),(1,1)$ in $A_{0}(S)$, we see that the entries of $D$ must satisfy the following conditions:

$$
\begin{gathered}
\max \left\{\left|a_{11}\right|,\left|a_{11} \pm a_{21}\right|,\left|a_{21}\right|\right\}=1, \quad \max \left\{\left|a_{12}\right|,\left|a_{12} \pm a_{22}\right|,\left|a_{22}\right|\right\}=1, \\
\max \left\{\left|a_{11}-a_{12}\right|,\left|a_{21}-a_{22}\right|,\left|\left(a_{11}-a_{12}\right) \pm\left(a_{21}-a_{22}\right)\right|\right\}=2, \\
\max \left\{\left|a_{11}+a_{12}\right|,\left|a_{21}+a_{22}\right|,\left|\left(a_{11}+a_{12}\right) \pm\left(a_{21}+a_{22}\right)\right|\right\}=2
\end{gathered}
$$

It is easy to verify that these conditions are compatible only if $\left|a_{11}\right|=\left|a_{22}\right|=1$ and $a_{12}=a_{21}=0$ or $\left|a_{12}\right|=\left|a_{21}\right|=1$ and $a_{11}=a_{22}=0$. Such $D$ 's are induced by homeomorphisms (that is, permutations) of $\partial_{e} S$ which can be extended to affine homeomorphisms of $S$ onto itself.

Finally, it should be noted that not all permutations $\sigma$ of $\partial_{e} S$ can be extended to affine homeomorphisms of $S$, the required condition for such an extension being

$$
\sigma\left(x_{1}\right)-\sigma\left(x_{2}\right)+\sigma\left(x_{3}\right)-\sigma\left(x_{4}\right)=0 .
$$

\section{Vector-valued case}

We now turn our attention to spaces of vector-valued functions. We first consider the space $C(X, E)$. The notion of a diameter-preserving map is clearly well defined in this situation. In the following theorem we consider a compact Hausdorff space $X$ and functions taking values in two different Banach spaces $E$ and $F$ and describe diameter-preserving linear bijections. Similar arguments hold when one considers different compact spaces in the domain. From now on we only consider vector spaces over the real scalar field.

Suppose $\phi$ is a homeomorphism of $X, S: E \rightarrow F$ a linear onto isometry and $T: C(X, E) \rightarrow F$ a linear operator. Define $\Phi: C(X, E) \rightarrow C(X, F)$ by $\Phi(f)(x)=$ $S(f(\phi(x)))+T(f)$ for $f \in C(X, E)$ and $x \in X$. Clearly, $\Phi$ is a diameter-preserving linear map.

In the vector-valued case one needs to assume some additional hypothesis (which is compatible with the scalar case) to ensure that $\Phi$ is a bijection. The following simple example illustrates this.

EXAMPLE 2. Let $\mathbb{R}^{2}$ denote the Euclidean plane. Consider $Z=\mathbb{R}^{2} \times \mathbb{R}^{2}$ equipped with the maximum norm (equivalently functions on a two point set with values in $\mathbb{B}^{2}$ ). Let $S=I$ be the identity map on $\mathbb{R}^{2}$ and let $T: Z \rightarrow \mathbb{R}^{2}$ be defined by $T(x, y)=-x$. Define $\Phi: Z \rightarrow Z$ by $\Phi(x, y)=(x, y)+(-x,-x)$. Clearly $\Phi$ is a diameter-preserving linear map. But $\Phi(x, x)=0$, so $\Phi$ fails to be one-to-one.

To remedy the above situation we now assume that $T$ is such that $T /(1 \otimes E)+S$ is a bijection of $E$ onto $F$ (for $e \in E, 1 \otimes e$ denotes the constant function $e$ on $X$ ). In 
the scalar case any $\Lambda \in A(K)^{\prime}$ such that $\Lambda(1) \neq-t$ clearly satisfies this condition. Thus this is compatible with the assumption made by [GM]. This condition is trivially satisfied if $T$ is such that $T /(1 \otimes E)=0$.

Suppose $\Phi(f)=0$. Since $S$ is an isometry and $\phi$ is a bijection, we get that $f$ takes the constant value $-S^{-1}(T(f))$. Hence since $S+T$ and $S$ are one-to-one, $f=T(f)=0$. Also for any $g \in C(X, F)$, let $f=S^{-1} \circ g \circ \phi^{-1}$. Let $e_{0} \in E$ be such that $T\left(1 \otimes e_{0}\right)+S\left(e_{0}\right)=T(f)$. Now let $h=f-\left(1 \otimes e_{0}\right)$. It is easy to see that $\Phi(h)=g$. Hence $\Phi$ is onto.

In order to obtain a complete description of diameter-preserving bijections, we need to impose an additional hypothesis on $E$ and $F$ that is analogous to the 'split face' condition imposed in the previous section.

THEOREM 2. Let $E$ and $F$ be Banach spaces such that the one-dimensional span of any extreme point of the dual unit ball is an L-summand. Let $\Phi: C(X, E) \rightarrow C(X, F)$ be a diameter preserving linear bijection. There exist an onto homeomorphism $\phi$ of $X$, an onto isometry $S: E \rightarrow F$ and a linear operator $T: C(X, E) \rightarrow F$, such that $T /(1 \otimes E)+S$ is a bijection and

$$
\Phi(f)(x)=S(f(\phi(x)))+T(f) \text { for } f \in C(X, E) \text { and } x \in X .
$$

ProOF. Let $\Phi: C(X, E) \rightarrow C(X, F)$ be any diameter preserving linear bijection. In what follows we will use the identification of $C(X, E)^{*}$ as $M\left(X, E^{*}\right)$, the space of $E^{*}$ valued countably additive regular Borel measures of finite variation equipped with the total variation norm. In this identification, it is well known that the extreme points of the unit ball of $M\left(X, E^{*}\right)$ can be identified with vector-valued Dirac measures $\delta(x) \otimes e^{*}$ for $x \in X$ and $e^{*} \in \partial_{e} E_{1}^{*}$. (For any Borel set $\mathrm{B},\left(\delta(x) \otimes e^{*}\right)(B)=e^{*}$ if $x \in B$ and $=0$ if $x \notin B, E_{1}^{*}$ denotes the unit ball of $E^{*}$.) Since our arguments will run parallel to those given in the case of scalar valued functions in [GU] we will only indicate briefly the corresponding steps. For $x \neq y$ and $e_{1}^{*} \neq \pm e_{2}^{*} \in \partial_{e} E_{1}^{*}$, since the measures are disjointly supported, and since line $\left\{e_{i}\right\}$ is an $L$-summand, we have,

$$
\left\|\delta(x) \otimes e_{1}^{*}+\delta(y) \otimes e_{2}^{*}\right\|=\left\|e_{1}^{*}\right\|+\left\|e_{2}^{*}\right\| .
$$

Therefore it is easy to see that for any $x, y \in X, x \neq y$ and $e^{*} \in \partial_{e} E_{1}^{*},(\delta(x)-$ $\delta(y)) \otimes e^{*}$ is an extreme point of the two-ball in the space of measures vanishing on 'constants'. Note that for any $f \in C(X, E)$

$$
\begin{aligned}
\operatorname{diam}(f(X)) & =\sup _{x, y \in X}\|f(x)-f(y)\|=\sup _{\substack{x, y \in X \\
e^{*} \in \theta_{\epsilon} E_{i}^{*}}}\left|e^{*}(f(x))-e^{*}(f(y))\right| \\
& =\sup _{\substack{x, y \in X \\
e^{*} \in \theta_{e} E_{i}^{*}}}\left|\left((\delta(x)-\delta(y)) \otimes e^{*}\right)(f)\right| .
\end{aligned}
$$


Thus as before $\{f \in C(X, E): \operatorname{diam}(f(X)) \leq 1\}^{\circ}=\overline{c o}\left\{(\delta(x)-\delta(y)) \otimes e^{*}: x, y \in\right.$ $X$ and $\left.e^{*} \in \partial_{e} E_{1}^{*}\right\}$.

We embed $E$ and $F$ into $C(X, E)$ and $C(X, F)$ respectively via the embeddings $e \rightarrow 1 \otimes e$ and $f \rightarrow 1 \otimes f(e \in E, f \in F)$ and consider the corresponding quotient spaces. The polar considered above is in the dual of these spaces.

Preserving our notation from Theorem 1 , it is now easy to see that there exists an onto homeomorphism $\phi$ of $X$ and a weak ${ }^{*}$-bi-continuous surjection $s: \partial_{e} F_{1}^{*} \rightarrow \partial_{e} E_{1}^{*}$ such that

$$
(\tilde{\Phi})^{*}\left(\delta\left(x_{0}\right)-\delta(x) \otimes f^{*}\right)=\left(\delta\left(x_{0}\right)-\delta(\phi(x))\right) \otimes s\left(f^{*}\right) .
$$

Using arguments similar to those given during the proof of Theorem 1, one can extend $s$ to an affine symmetric weak*-homeomorphism from $F_{1}^{*}$ onto $E_{1}^{*}$, still denoted by $s$. A routine exercise involving the Banach-Dieudonné theorem (see [H, page 154]) gives an onto isometry $S: E \rightarrow F$ such that $S^{*}$ agrees with $s$ on $F_{1}^{*}$.

By going back from the quotient spaces to the original function spaces, we get a linear operator $T: C(X, E) \rightarrow F$, such that $T /(1 \otimes E)+S$ is a bijection and $\Phi(f)(x)=S(f(\phi(x))+T(f)$ for $f \in C(K, E)$ and $x \in X$.

We now give an example of a discontinuous linear map $T$ satisfying the 'necessary' condition of the above theorem.

Example 3. Let $E=F=c_{0}$. Let $X$ be any infinite compact Hausdorff space. Consider the identity homeomorphism on $X$. Let $S=I$. Let $\left\{e_{n}\right\}_{n \geq 1}$ be the canonical Schauder basis in $c_{0}$. Consider an extension of it to a Hamel basis. Let $T^{\prime}: c_{0} \rightarrow c_{0}$ be the unbounded linear map given by $T^{\prime}\left(e_{n}\right)=(n-1) e_{n}$ for all $n$ and $T^{\prime}$ is zero at other basis vectors. Consider the embedding of $c_{0}$ into $C\left(X, c_{0}\right)$, via, $e \rightarrow 1 \otimes e$. Let $P$ denote a canonical projection from $C\left(X, c_{0}\right)$ onto this image (evaluation at a fixed point of $X)$. Let $T=T^{\prime} \circ P$. We claim that $S+T /\left(1 \otimes c_{0}\right)$ is a linear bijection. It is clearly enough to verify this at a canonical vector $e_{k}$. If $S\left(e_{k}\right)+T\left(1 \otimes e_{k}\right)=0$, then $T^{\prime}\left(e_{k}\right)=-e_{k}$ gives the necessary contradiction. The equation $(1 / k) e_{k}+T^{\prime}(1 / k) e_{k}=$ $e_{k}$ shows that $S+T /\left(1 \otimes c_{0}\right)$ is onto.

Another space of vector-valued functions for which our method of proof can be made to work is the space $A(K, E)$ of $E$-valued affine continuous functions defined on a compact convex set $K$, equipped with the supremum norm. When $K$ is a Choquet simplex, it follows from the results of Ruess [Ru] (since the space $A(K)$ has the approximation property) that $A(K, E)$ can be identified with the injective tensor product space $A(K) \otimes_{\varepsilon} E$ (we recall here the well known identification of $C(X, E)$ as $C(X) \otimes_{\varepsilon} E$, see [DU, Chapter VIII]). Since any $k \in \partial_{e} K$, is a split face, it follows from the results of Ruess and Werner [RW] (see also [R2]) that for $k_{1}, k_{2}, \in \partial_{e} K, k_{1} \neq k_{2}$ and for any $e^{*} \in \partial_{e} E_{1}^{*},\left\|\delta\left(k_{1}\right) \otimes e^{*}-\delta\left(k_{2}\right) \otimes e^{*}\right\|=2$ (for any $a \in A(K, E)=A(K) \otimes_{\varepsilon} E$, 
$\left.\left(\delta(k) \otimes e^{*}\right)(a)=e^{*}(a(k))\right)$. Thus the following proposition can be proved following arguments indicated before.

PROPOSITION 2. Let $E$ and $F$ be Banach spaces such that the one-dimensional span of any extreme point of the dual unit ball is an L-summand. Let $K$ be a Choquet simplex. Let $\Phi: A(K, E) \rightarrow A(K, F)$ be a diameter preserving linear bijection. Then there exists an onto affine homeomorphism $\phi$ of $K$, an onto isometry $S: E \rightarrow F$ and a linear map $T: A(K, E) \rightarrow F$, such that $S+T / 1 \otimes E$ is a bijection and

$$
\Phi(a)(k)=S(a(\phi(k)))+T(a) \quad \text { for } a \in A(K, E) \text { and } k \in K .
$$

As a final application of our methods we consider $W^{*} C\left(X, E^{*}\right)$, the space of functions that are continuous when $E^{*}$ has the weak ${ }^{*}$ topology, equipped with the supremum norm. Here again for any onto homeomorphism $\phi$ of $X$, for any onto isometry $S$ of $E$ and for any linear map $T: W^{*} C\left(X, E^{*}\right) \rightarrow E^{*}$, such that $S^{*}+T$ is a bijection on $1 \otimes E^{*}$,

$$
\Phi(f)=S^{*}(f(\phi(x)))+T(f)
$$

for $f \in W^{*} C\left(X, E^{*}\right)$ and $x \in X$, is a diameter-preserving linear bijection.

It is well known and easy to see that this space of functions can be isometrically identified with the space of bounded operators $\mathscr{L}(E, C(X))$, via the map $f \rightarrow f^{\wedge}$, where $f^{\Lambda}: E \rightarrow C(X)$ is defined by $f^{\Lambda}(e)=e^{\Lambda} \circ f$ for $f \in W^{*} C\left(X, E^{*}\right)$ and $e \in E$ ( $e^{\wedge}$ denotes the evaluation functional on $E^{*}$ ). When $X$ is the Stone-Čech compactification of a discrete set, this function space can be identified with the $\ell^{\infty}$. direct sum of copies of $E^{*}$.

For the remainder of this paper we consider $E$ as a subspace of $E^{* *}$ under the canonical embedding. We call an extreme point of the unit ball of $E$ a weak $k^{*}$-extreme point, if it is an extreme point of the unit ball of $E^{* *}$. In the following proposition we assume that the unit ball of $E$ is the closed convex hull of its weak*-extreme points. It follows from [DU, Theorem 3, Chapter VII], that if $E$ has the Radon-Nikodym property, then this hypothesis is satisfied. The space $\ell^{\infty}$ and $\mathscr{L}\left(\ell^{2}\right)$ are some of the other examples of spaces that satisfy this condition (see [R3]). It is well known that for any compact Hausdorff space $Y$, every extreme point of the unit ball of $C(Y)$ is a weak ${ }^{*}$-extreme point and thus if $Y$ in addition is totally disconnected then the unit ball is the norm closed convex hull of its weak*-extreme points.

PROPOSITION 3. Let $X$ be a compact Hausdorff space with a dense set of isolated points. Let $E$ be a Banach space such that the unit ball is the closed convex hull of its weak $k^{*}$-extreme points and such that the one-dimensional span of weak $k^{*}$-extreme point is an $L$-summand. Then any diameter-preserving bijection of $W^{*} C\left(X, E^{*}\right)$ is of the form described above. 
PROOF. Let $I$ denote a dense set of isolated points of $X$. Let $J$ denote the set of weak*-extreme points. For any $f \in W^{*} C\left(X, E^{*}\right)$,

$$
\begin{aligned}
\operatorname{diam}(f(X)) & =\{\|f(x)-f(y)\|: x, y \in X\} \\
& =\{|e(f(x))-e(f(y))|: x, y \in I, e \in J\} .
\end{aligned}
$$

For any $x \in X$ and $e \in E$, let $\delta(x) \otimes e$ denote the functional $(\delta(x) \otimes e)(f)=f(x)(e)$. Since $x$ is an isolated point and $e$ is an extreme point of the unit ball of the bidual, it follows from [Li, Theorem 3.7] that $\delta(x) \otimes e$ is an extreme point of the unit ball of $W^{*} C\left(X, E^{*}\right)^{*}$. Furthermore, for any distinct $x, y \in I$, it can be shown that $(\delta(x)-\delta(y)) \otimes e$ is an extreme point of the two-ball of functionals vanishing on 'constants'. The rest of the arguments needed to reach the desired conclusion are identical to the ones given before.

After completing our work on this paper we have received from Professor Felix Cabello Sanchez a preprint of his paper 'Diameter preserving linear maps and isometries (II)', where he has obtained by different methods, a description of diameter-preserving linear bijections of $A(K)$, when $K$ is a Choquet simplex.

Note added on 10-10-2000. The above preprint has since appeared in Proc. Indian. Acad. Sci. (Math. Sci.) 110 (2000) 205-211. Since this paper was written the question raised in Remark 1 has been settled in the negative and will be the basis of some future work in this area.

\section{Acknowledgement}

When this work was done (1999) the first author was a Visiting Professor at the University of Missouri-Columbia. He is grateful to Professor N. J. Kalton, Professor E. Saab and their colleagues for their warm hospitality.

\section{References}

[AE] L. Asimow and A. J. Ellis, Convexity theory and its applications in functional analysis, London Math. Soc. Monographs 16 (Academic Press, London, 1980).

[B] E. Behrends, $M$-structures and the Banach-Stone theorem, Lecture Notes in Math. 736 (Springer, Berlin, 1979).

[C] F. Cabello Sanchez, 'Diameter preserving linear maps and isometries', Arch. Math. 73 (1999), 373-379.

[D] J. Diestel, Sequences and series in Banach spaces, Graduate Texts in Math. 92 (Springer, Berlin, 1984).

[DU] J. Diestel and J. J. Uh1, Vector measures, Amer. Math. Soc. Surveys 15 (Providence, RI, 1977). 
[ES] A. J. Ellis and W. S. So, 'Isometries and complex state spaces of uniform algebras', Math. Z. 195 (1987), 119-125.

[FJ] R. J. Fleming and J. E. Jamison, 'Isometries on Banach spaces: A survey', in: Analysis, geometry and groups: A Riemann legacy volume (Hadronic Press, Palm Harbor, 1993) pp. 52-123.

[GJ] L. Gillman and M. Jerison, Rings of continuous functions, Graduate Texts in Math. 43 (Springer, Berlin, 1976).

[GU] F. González and V. V. Uspenskij, 'On homeomorphisms of groups of integer-valued functions', Extracta Math. 14 (1999), 19-29.

[GM] M. Györy and L. Molnar, 'Diameter preserving linear bijections of $C(X)$ ', Arch. Math. 71 (1998), 301-310.

[H] R.B. Holmes, Geometric functional analysis and its applications, Graduate Texts in Math. 24 (Springer, Berlin, 1975).

[L] A. J. Lazar, 'Affine products of simplexes', Math. Scand. 22 (1968), 165-175.

[Li] Å. Lima, E. Oja, T. S. S. R. K. Rao and D. Werner, 'Geometry of operator spaces', Michigan Math. J. 41 (1994), 473-490.

[R1] T. S. S. R. K. Rao, 'Isometries of $A_{\mathrm{C}}(K)$ ', Proc. Amer. Math. Soc. 85 (1982), 544-546.

[R2] _ _ ' $M$-structure and the space $A(K, E)$ ', Rend. Mat. Appl. 15 (1995), 153-160.

[R3] _ - 'Denting and strongly extreme points in the unit ball of spaces of operators', Proc. Indian Acad. Sci. (Math. Sci.) 109 (1999), 75-85.

[Ru] W. Ruess, 'Duality and geometry of spaces of compact operators', in: Surveys and recent results III. Proc. Pederborn Conference on Functional Analysis (eds. K. Bierstedt and B. Fuchssteiner), North-Holland Math. Studies 90 (North-Holland, Amsterdam, 1984) pp. 59-78.

[RW] W. Ruess and D. Werner, 'Structural properties of operator spaces', Acta Univ. Carol. Math. Phys. 28 (1987), 127-136.

[W] R. C. Walker, The Stone-Čech compactification, Ergebnisse der Mathematik und ihrer Grenzgebiete Band 83 (Springer, Berlin, 1974).

Statistics and Mathematics Unit

Indian Statistical Institute

R.V. College Post

Bangalore 560059

India

e-mail: tss@isibang.ac.in
Statistics and Mathematics Unit Indian Statistical Institute 203 B.T. Road Calcutta 700035 India e-mail: ashoke@isical.ac.in 
